\title{
Comparative chromosome painting discloses homologous segments in distantly related mammals
}

\author{
Harry Scherthan ${ }^{1}$, Thomas Cremer ${ }^{2}$, Ulfur Arnason ${ }^{3}$, Heinz-Ulrich Weier ${ }^{4}$, \\ Antonio Lima-de-Faria ${ }^{3} \&$ Lutz Frönicke ${ }^{1}$
}

Comparative chromosome painting, termed ZOO-FISH, using DNA libraries from flow sorted human chromosomes 1, 16, 17 and $X$, and mouse chromosome 11 discloses the presence of syntenic groups in distantly related mammalian orders ranging from primates (Homo sapiens), rodents (Mus musculus), even-toed ungulates (Muntiacus muntjak vaginalis and Muntiacus reevesi) and whales (Balaenoptera physalus). These mammalian orders have evolved separately for 55-80 million years (Myr). We conclude that ZOO-FISH can be used to generate comparative chromosome maps of a large number of mammalian species.

'Division of Human Biology and Human Genetics, ErwinSchroedingerstrasse, D-67663

Kaiserslautern, Germany

${ }^{2}$ Institute of Human Genetics and

Anthropology, Im

Neuenheimer Feld

$328, D-69120$

Heidelberg,

Germany

${ }^{3}$ Institute of

Genetics, University

of Lund, Solvegatan

29, S-223 62 Lund,

Sweden

'Division of

Molecular

Cytometry,

MCB230,

Department of

Laboratory

Medicine,

University of

California at San

Francisco, San

Francisco,

Califormia 94143-

0808, USA

Correspondance should be addressed to H.S.
Comparative cytogenetics has provided a powerful tool to establish interspecific chromosome homologies, but the interpretation of banding patterns has met with difficulties in families with rapid karyotype evolution, such as gibbons, or in distantly related species ${ }^{1,2}$. Comparative gene mapping has made possible the generation of comparative physical maps of 28 species representing different mammalian orders ${ }^{3}$. However, this approach is laborious and time consuming. Accordingly, the resolution of present maps is still strongly limited in most species due to the low number of comparatively mapped loci ${ }^{3,4}$. Recently it was demonstrated that chromosome painting using composite DNA probes established from flow sorted human chromosomes is a valuable tool to elucidate karyotype rearrangements in primate evolution ${ }^{5-10}$. However, up to now attempts to extend this approach to other mammalian orders were not successful, although it was not clear whether this limitation was due to DNA sequence diversity between distantly related species or to inadequacies of the available protocols ${ }^{11,12}$.

In the present study we describe an improved protocol, termed ZOO-FISH, for comparative chromosome painting, that is capable of detecting homologous chromosome segments in species representing different mammalian orders (primates, rodents, even-toed ungulates and whales). For this purpose, plasmid library DNA probes ${ }^{13}$, as well as linker adaptor library DNA probes ${ }^{14}$ established from flow sorted human and mouse chromosomes were labelled with biotin or digoxigenin. The composite DNA probes were hybridized to metaphase spreads of evolutionarily distant species together with an excess of unlabelled Cot1-DNA fractions from the species from which the DNA library was established, that is, human or mouse. For a successful comparative painting experiment three parameters were found to be most important. Namely, the suitability of the chromosome spreads for ZOO-FISH, an increased probe concentration and a prolonged hybridization time. The probe concentration and hybridization time were modified on the assumption that the fraction of probe sequences which closely match the chromosome target sequences would diminish when library DNA probes from a given species were hybridized to metaphase spreads from distantly related species. From previous experiments it was known that a probe complexity representing some $2 \%$ of the sequences contained in a microdissected human chromosome fragment was sufficient for appropriate painting of this segment in human metaphase spreads ${ }^{15}$. Accordingly, it could be expected that a few percent of highly conserved probe and target sequences should be sufficient to generate appropriate signals in ZOO-FISH experiments. This approach was successfully applied to generate fluorescence signals at segments of synteny in metaphase spreads from human, mouse (Mus musculus), Indian muntjac (Muntiacus muntjak vaginalis), Chinese muntjac (M. reevesi) and fin whale (Balaenopteraphysalus). The species investigated reflect evolutionary distances of about 3-10 Myr between the two muntjac species ${ }^{16}$, about $55 \mathrm{Myr}$ between even-toed ungulates (Artiodactyla) and whales and more than $80 \mathrm{Myr}$ between any other combination of species ${ }^{17}$. Using optimal chromosome preparations and hybridization protocols, $20-70 \%$ of the evaluated metaphase spreads showed the specific hybridization patterns. In all experiments signal intensities 


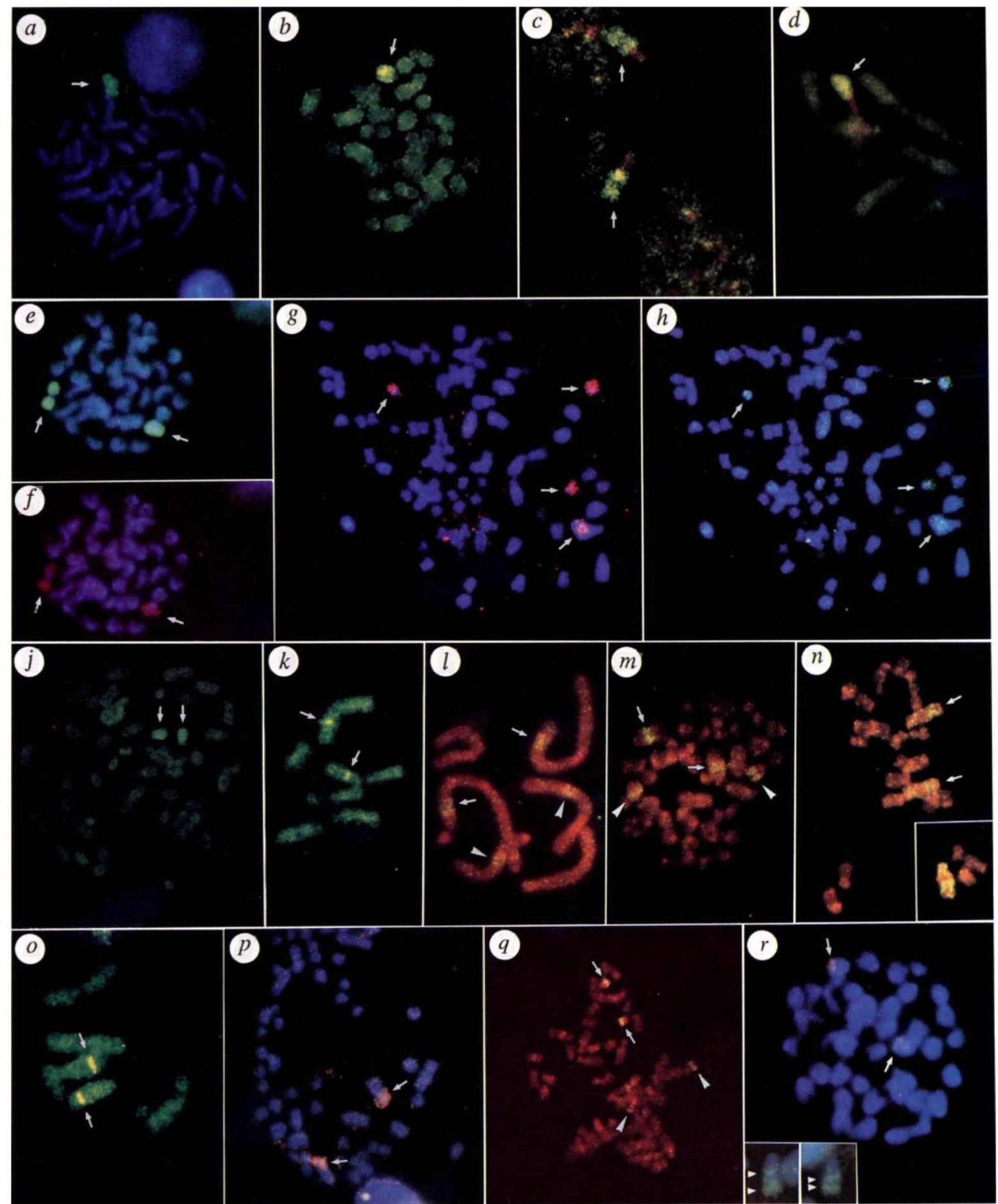




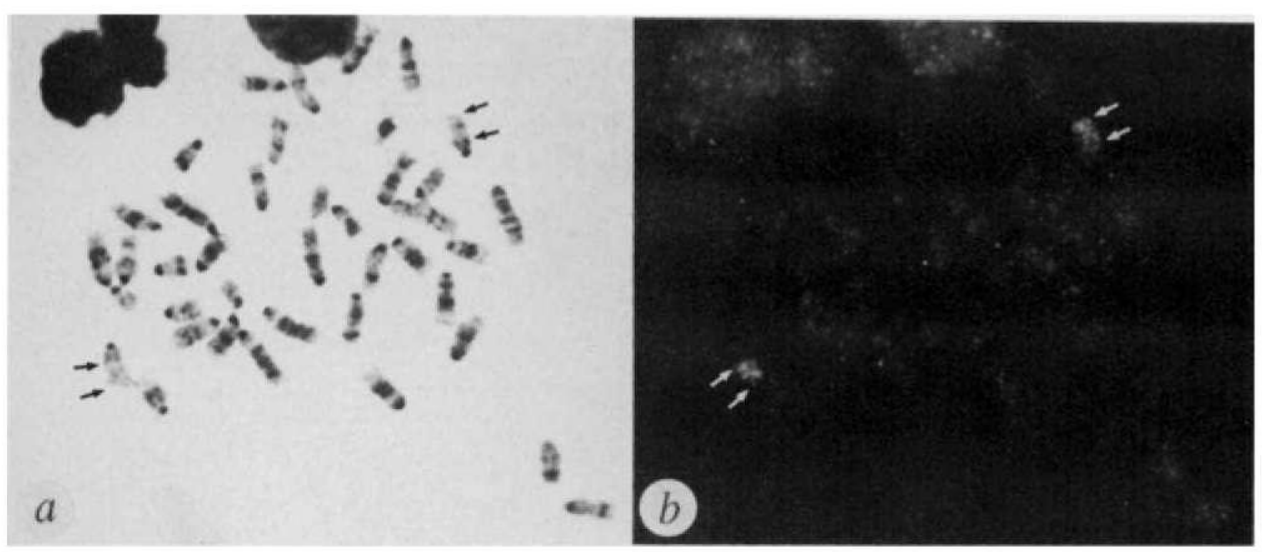

Fig. 2 a, GTG banded mouse metaphase spread. $b$, The same spread subjected to ZOO-FISH with human chromosome 17 specific library DNA shows a painted segment on chromosome 11 at band $B-E^{51}$ (arrows).

were sufficient for evaluation by standard epifluorescence microscopy and microphotography.

\section{Identification of syntenic segments}

A series of model experiments was undertaken to test the efficiency of ZOO-FISH as a tool to map karyotypic changes during the evolution of mammals and to assess the conservation of syntenic groups between distantly related mammals. In accord with Ohno's hypothesis ${ }^{18,19}$ the human X-chromosome composite probe ${ }^{14}$ hybridized across the whole $\mathrm{X}$-chromosome, except for the constitutive heterochromatic regions, in human (not shown), mouse, Chinese muntjac and fin whales (Fig. $1 a-$ c). This observation confirms that the mammalian $\mathrm{X}$ chromosome has been highly conserved during the evolution of the Eutheria. In the Indian muntjac Xchromosome, which is much larger than in the other species, painting was restricted to the short arm (Fig. 1d). This finding supports other observations indicating that the Indian muntjac $\mathrm{X}$ chromosome evolved by a translocation of an ancestral X of the Cervidae and a large autosomal part ${ }^{20,21}$. This interpretation is consistent with the $\mathrm{X} / \mathrm{Y}_{1}, \mathrm{Y}_{2}$ mechanism proposed by $\mathrm{Ohno}^{18}$ for species with X-autosome translocations. Painting experiments with the human X-chromosome specific composite probe resulted in rather homogeneous painting of the Xhomologue in all species tested so far.

From comparative mapping experiments it is known that extended homologies exist between human chromosome 17 and mouse chromosome 11 (ref. 22). As expected, ZOO-FISH using the human chromosome 17 specific composite DNA probe and GTG-banded mouse metaphase spreads demonstrated specific painting of mouse chromosome 11 B-E (Fig. 2a,b). Two-colour ZOOFISH to mouse metaphase spreads with both mouse chromosome 11 and human chromosome 17 specific composite DNA probes resulted in simultaneous painting of mouse chromosome 11 (Fig.1e,f). Also, two-colour painting with this probe combination on human metaphase spreads detected human chromosome 17 with both the mouse chromosome 11 specific probes (Fig. 1j) and the human probes (not shown). Potential chromosome homologies of human chromosome 17 and mouse chromosome 11 and the karyotypes of the deer and the whale have not been established so far. Notably, two-colour painting experiments, with both chromosome libraries, to metaphase spreads from the fin whale revealed co-localization of the hybridization signals on two pairs of chromosomes (Fig. $1 g, h$ ). A single segment was painted with the mouse chromosome 11 composite probe on the chromosome 1 of the Indian muntjac (Fig. $1 k$ ).

Painting with a human chromosome 1 composite probe and Indian muntjac metaphase spreads revealed the presence of a syntenic segment on chromosome 1 and another syntenic segment on the autosomally derived long arm of the $\mathrm{X}$ and its homologue, $\mathrm{Y}_{1}$ (Figs $1 h$ 2).

Fig. 14 Extent of the syntenic chromosome segments as demonstrated by ZOO-FISH in metaphase spreads from human, mouse (Mus musculus), Indian muntjac, Chinese muntjac and fin whale. ZOO-FISH of the euchromatic part of the X-chromosomes (arrows) of: $a$, the mouse, $b$, the Chinese muntjac, $c$, the fin whale and $d$, the Indian muntjac using human X-chromosome specific library DNA as the probe. Two-coiour painting of mouse chromosome 11 obtained with; $e$, biotin labelled mouse chromosome 11 library DNA ${ }^{50}$ (detected by avidin-FITC; green, arrows) and $f$, digoxigenin labeled human chromosome 17 library DNA (detected by Cy3; red, arrows) hybridized simultaneously to metaphase chromosomes of Mus musculus. Two colour-painting observed on two small sized fin whale chromosomes after ZOO-FISH with; $g$, digoxigenin labelled human chromosome 17 library DNA (detected by Cy3; red, arrows) and; $h$, biotin labelled mouse chromosome 11 library DNA (detected by avidin-FITC; green, arrows). $j$, ZOO-FISH with mouse chromosome 11 library DNA on chromosome 17 in a human metaphase spread (arrows). Identification of chromosome 17 was achieved by simultaneous painting with human chromosome 17 library DNA (not shown). $k$, A single interstitial segment was delineated on chromosome 1 of the Indian muntjac (arrows) with the mouse chromosome 11 composite probe. ZOO-FISH with human chromosome 1 library DNA showed, I, two interstitial segments, one on Indian muntjac chromosomes 1 (arrows), and one on chromosome $X$ and $Y_{1}$ (arrow heads). $m$, The chromosome 1 composite probe delineated an interstitial segment in a large chromosome (arrows) and the distal part of a small chromosome (arrow heads) in a Chinese muntjac metaphase spread and $n$, an entire chromosome pair shown in a partial metaphase spread from the fin whale (arrows). The inset shows the same painted fin whale chromosome from another metaphase spread. ZOO-FISH with human chromosome 16 library DNA showing; 0 , a segment of Indian muntjac chromosome 2 (arrows); $p$, the distal half of a large chromosome in a Chinese muntjac metaphase spread (arrows); $q$, The euchromatic part of an acrocentric, small chromosome in the fin whale except for the centromeric satellite DNA (arrows). A smaller signal was aiso observed at another small chromosome pair (arrow heads). $r$, A distal segment of mouse chromosome 8 was delineated in a metaphase spread of Mus musculus. The inset shows more elongated chromosomes exhibiting two distinct, closely spaced painted segments. Chromosome identification was achieved by DAPI banding prior to ZOO-FISH (not shown). Microphotographs were recorded on colour slide film using conventional fluorescence microscopy. Panels $a, d, e, f, g, h, p$ and $r$ show double exposures of signals together with DAPI; $b, k$ and 0 show FITC fluorescence only; $a, c, l, m, n$ and $q$ show double exposures of signals with propidium iodide. 
Similarly, two syntenic chromosome segments were identified in the Chinese muntjac. One segment is located interstitially in a large chromosome, while the other segment maps to the distal part of a medium sized chromosome (Fig. $1 \mathrm{~m}$ ). In contrast, homology with a single large chromosome was disclosed in fin whale metaphase spreads (Fig. $1 n$ ).

ZOO-FISH with the human chromosome 16 composite probe and Mus musculus metaphase spreads revealed the presence of a homologous distal segment in mouse chromosome 8 (Fig. 1 $r$ ). In this experiment chromosome identification was performed by DAPI-banding prior to FISH (not shown). In agreement with published comparative maps $\mathrm{s}^{22}$ mouse chromosomes 8 occasionally revealed two closely spaced painted segments separated by a non painted segment (Fig. lr, insert). Published data $^{22}$ indicate an approximate size of the these segments between 7 and $18 \mathrm{Mbp}$. Other still smaller segments $(\leq 5$ Mbp) with homology to human chromosome 16 have been described for mouse chromosomes 7,11,16 and 17 (ref. 22), but these could not be identified by ZOO-FISH.

ZOO-FISH with the human chromosome 16 composite probe and metaphase spreads of the Chinese and Indian muntjac revealed an extended homologous segment in the chromosome 2 of the two Muntjac species (Fig. 1o,p). The chromosomal location of the painted segments is in agreement with previous banding data indicating homology between the distal part of chromosome 2 of the Chinese muntjac with an internal segment of chromosome
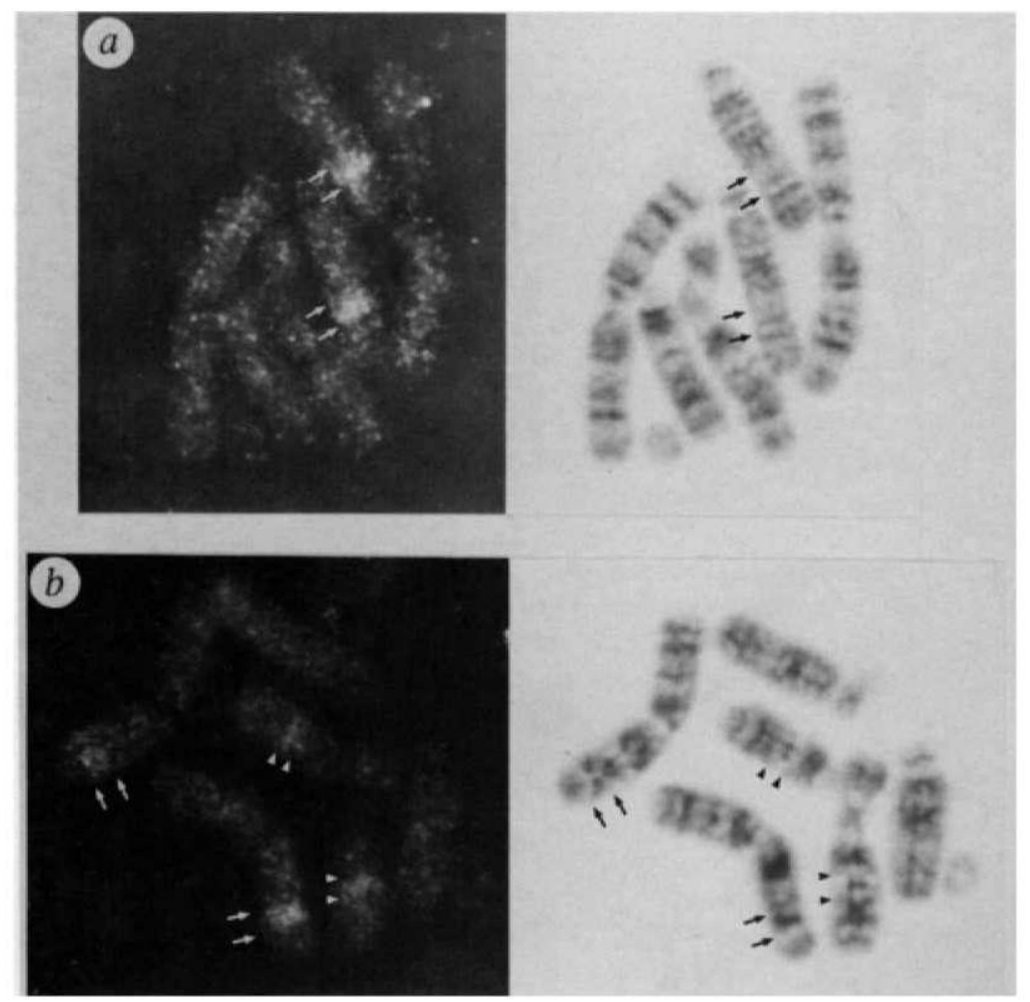

Fig. $3 a$, ZOO-FISH with human chromosome 16 and $b$, Human chromosome specific library DNA to GTG banded chromosomes of the Indian muntjac ${ }^{26}$. The chromosome 16 library DNA produced a painted segment on chromosome 2 at band 2q26-32 (a, arrows) and the human chromosome 1 specific library DNA delineated two segments, one at chromosome 1q26-31 (b, arrows) and one on the long arm of the $X$ chromosome and its homologous counterpart $Y 1$ at band q21-32 (b, arrowheads).
2 of the Indian muntjac ${ }^{21}$. Fin whale metaphase spreads hybridized with the human chromosome 16 composite probe showed strong signals at the euchromatic part of a small chromosome pair, while a smaller signal could be detected on another small chromosome pair (Fig. 1q).

To investigate whether synteny is maintained in both the apparently gene poor GTG-positive bands and the more gene rich GTG-negative bands ${ }^{23,24}$ we performed comparative chromosome painting with the human chromosome 1 and 16 libraries on GTG-banded Indian muntjac chromosomes (Fig. 3). These experiments, as well as the painting of mouse chromosome 11 with human chromosome 17 composite probes (Fig. 2) revealed a rather homogeneous painting of chromosome segments containing G-band positive and G-band negative chromatin (see Discussion).

Human chromosomes 1 and 16 comprise a DNA fraction of $8.0 \%$ and $3.0 \%$, respectively, of the male human genome ${ }^{25}$. These data are in reasonable agreement with the relative DNA contents of $7.5 \%$ and $2.8 \%$, respectively, estimated for the chromosome segments painted by the human chromosome 1 and 16 composite probes in metaphase spreads from a male Indian Muntjac ${ }^{26}$. To obtain an estimate of the genome fraction painted on mouse chromosome 11 with the human chromosome 17 composite probe we determined the relative length of the painted segments $(2.9 \%)$ as a fraction of the total length of all chromosomes from several male metaphase spreads. The same value was recorded for chromosome 17 in male human metaphase spreads ${ }^{25}$. These data suggest that ZOO-FISH illuminated chromosome segments comprise similar genomic fractions in species from various mammalian orders.

\section{Discussion}

ZOO-FISH with human whole chromosome composite probes can serve as an efficient tool to identify chromosome segments which have maintained synteny during evolution. The size of the smallest syntenic segment which could be detected in the present experiments was approximately $7 \mathrm{Mb}$. It should be noted that evaluation was restricted to signals which could be unequivocally identified by conventional fluorescence microscopy and documented by colour slide film. The use of digital fluorescence microscopy with sensitive $C C D$ cameras in combination with multicolour FISH $^{27-30}$ should help to further increase the resolution and speed with which comparative chromosome maps can be established. We expect, however, that even with more sophisticated evaluation the smallest syntenic segments which can be detected using whole chromosome painting probes will remain in the order of a few megabases. This would correspond to the smallest trans- 
located segments which have been decteted in human metaphase spreads using whole chromosome paint probes. Besides DNA libraries established from flow sorted chromosomes of various species ${ }^{13,14,31,32}$, microdissection libraries ${ }^{7,15,33}$, probe contigs which span chromosome segments of interest and probes defining individual coding gene loci (type I anchor loci) ${ }^{4}$ can be used in multicolour ZOO-FISH schemes. In this way the overall or local resolution of the comparative chromosome map from each species can be adjusted to the needs of each investigation.

A rapid generation of comparative maps from species of all mammalian orders will facilitate the reconstruction of ancient karyotypes for each order. ZOO-FISH also provides a powerful tool to test models for karyotype evolution ${ }^{34-36}$. Synteny of certain chromosome segments between two current species may simply indicate that the number of viable chromosomal rearrangements after their evolutionary separation were not sufficient to separate these segments. Alternatively, and more interestingly, syntenic segments pinpoint genomic regions whose integrity has been maintained during evolution by selective pressure. In order to speed up the search for syntenic chromosome segments in distantly related species we are presently trying to extend ZOO-FISH with human whole and subregional chromosome paint probes to other vertebrate classes. The realization of such an approach should be strongly facilitated using composite probes enriched for conserved sequences ${ }^{37}$.

Wienberg et al. ${ }^{5}$ have reported that FISH with a human $\mathrm{X}$-chromosome composite probe yielded rather uniform painting of hominoid $\mathrm{X}$ chromosomes, while an R-bandlike hybridization pattern was seen in lower primates. In the present experiments such a distinction could not be observed for more distantly related mammalian species. A possible correlation of their finding was suggested with data indicating that $\mathbf{R}$-bands contain more conserved DNA sequences than G-bands $s^{23,24,36}$. It is currently not clear whether ZOO-FISH provides a suitable approach to explore differences in the relative contents of conserved sequences along individual chromosomes. The recently introduced technique of comparative genome hybridization $(\mathrm{CGH})^{38-40}$ may help to improve the sensitivity of ZOO-FISH in detecting such differences. $\mathrm{CGH}$ of the genomic DNAs of two evolutionary distant mammalian species $A$ and $B$ to metaphase spreads of one species would allow fluorescence to measure quotients in $\mathrm{G}$ and R-bands. A lower conservation of sequences in $\mathrm{G}$ bands, compared to R-bands, should then be paralleled by corresponding differences of the fluorescence quotients.

In addition to the study of chromosome evolution, ZOO-FISH has important implications for livestock genome research ${ }^{41}$ and the cloning of genes responsible for inherited diseases or the development of tumors in model animals.

Mapping a disease gene of interest to a certain chromosomal segment may in future be facilitated by improved protocols for genomic mismatch scanning which allow linkage analysis in the absence of informative markers ${ }^{42}$. The generation of a sufficiently detailed comparative chromosome map for the species in question would allow identification of the homologous chromosome segment in human or mouse, two species for which highly resolved genetic maps already exist. Such an approach could immediately provide information on genes mapped in the syntenic segment of interest and thus assist in positional cloning in species in which only fragmentary comparative map exists.

It has been demonstrated that $\mathrm{CGH}$ allows the rapid mapping of recurrent gains and losses of chromosomes and chromosomal subregions ( $\geq 10 \mathrm{Mbp}$ ) in tumour genomes $^{38-40}$. CGH performed with tumour DNAs from each species to their normal chromosome complements will disclose for each species a set of recurrent gains and losses. If the genetic mechanism(s) involved in the development of the tumour entity in question were identical in both species, the two sets should carry homologous chromosomal segments harboring the same tumour relevant genes, ZOO-FISH can be used to demonstrate and specify such homologous segments unequivocally. In this way ZOO-FISH not only provides an important tool to establish whether a tumour studied in an animal provides a reasonable model for a human clinical tumour but will also help to narrow down commonly involved regions prior to subsequent positional cloning of relevant genes.

\section{Methodology}

Cell lines and chromosome preparation. Cell lines of a male Indian (Muntiacus muntjacus vaginalis) and a male Chinese muntjac ( $M$. reevesi) were cultured and harvested as described ${ }^{16}$. Chromosome preparations of Mus musculus (strain C57BL) were obtained from lipopolysaccharide stimulated spleen cells (H.-U.W, unpublished data). Spreads from male $(102 / \mathrm{El} \times \mathrm{C} 3 \mathrm{H} / \mathrm{El})$ mice $^{43}$ were kindly provided by G. Schriever-Schwemmer, (GSF, Institut fúr Säugertiergenetik, Neuherberg, Germany). Fin whale (Balaenoptera physalus) chromosomes were prepared from fibroblast cell lines ${ }^{44}$. Human chromosome spreads were prepared from PHA stimulated peripheral blood lymphocytes. For all chromosome preparations a standard protocol was applied including hypotonic treatment with $0.0375 \mathrm{M} \mathrm{KCl}$ and fixation $(6 \times)$ with acetic acid/methanol (3:1) freshly prepared for each step. Special care was taken to obtain cytoplasm free chromosome preparations. A crisp appearance of the chromosomes in phase contrast and intensive staining with DNA specific fluorochromes, such as DAPI, are good indications of their usefulness in ZOO-FISH experiments. However, the actual usefulness of individual chromosome preparations can only be verified by experiment and it may become necessary to prepare and test several batches of chromosome spreads. GTG-banding of chromosome spreads prior to ZOO-FISH was performed as described ${ }^{45}$ with the modification that the embedding of the GTG-banded metaphase spreads in Eukitt was omitted.

DNA probes and labelling. DNA from the human chromosome specific plasmid libraries ${ }^{13}$ was prepared and nick-translated with biotin-14-dATP (Life Technologies, Gaithersburgh) or digoxigenin11-dUTP (Boehringer Mannheim) using a nick translation kit (Life Technologies). DNA from the human chromosome X specificlinker adaptor library ${ }^{14}$ and the mouse chromosome 11 specific linker adaptor library was amplified by PCR and labeled with biotin-11dUTP (Sigma) as described ${ }^{14}$. In all cases the final size of probe fragments was adjusted to $100-300$ bp by DNase I digestion.

In situ hybridization. To obtain specific painting of chromosomal segments in distantly related mammals it was necessary to modify standard protocols of chromosomal in situ suppression (CISS)hybridization ${ }^{1,12}$. In the case of the plasmid libraries with human inserts, probe concentration in the hybridization solution $(50 \%$ formamide, $2 \times$ SSC, $10 \%$ dextran sulfate) was increased to $330 \mathrm{ng} \mathrm{\mu l}^{-1}$ (as compared to concentrations of 2-10 $\mathrm{ng} \mu^{-1}$ routinely used for CISS-hybridization of these libraries to human metaphase spreads ${ }^{\mathrm{t3}}$ ). For the mouse linker adaptor library optimal experimental results were obtained at $200 \mathrm{ng} \mu^{-1}$. Cotl DNA (Life technologies) from human or mouse (depending on origin of the chromosome specific libraries) was added to the hybridization mixture (final concentration $2 \mu \mathrm{g} \mu \mathrm{t}^{-1}$ ) as some repetitive DNA motives are shared among evolutionary distant species ${ }^{46,47}$. In two-colour chromosome painting 
experiments using human and mouse composite probes simultaneously both human and mouse Cotl DNA was added in equal amounts (final concentration $1 \mu \mathrm{g} \mathrm{hl}^{-1}$ each). Hybridization mixture and chromosome spreads were sealed under a coverslip and denatured simultaneously for $3 \mathrm{~min}$ at $75^{\circ} \mathrm{C}$ on a hot plate. After hybridization for $72 \mathrm{~h}$ at $37^{\circ} \mathrm{C}$ preparations were washed $3 \times 5 \mathrm{~min}$ in $0.05 \times \mathrm{SSC}$ and blocked for $5 \mathrm{~min}$ in BT buffer (BT $=0.15 \mathrm{M}$ $\mathrm{NaHCO}_{3}, 0.1 \%$ Tween $20, \mathrm{pH} 8.3$ ) with $0.05 \%$ BSA. Biotinylated hybrid molecules were detected with avidin-FITC (Sigma) and one round of signal amplification ${ }^{48}$. The digoxigenin labelled probe molecules were detected with $\mathrm{Cy} 3$ conjugated secondary and tertiary antibodies (Jackson Immuno Research) to an anti-dig mouse monoclonal antibody (Boehringer) ${ }^{49}$. Finally chromosome spreads were counterstained with 4,6-diamino-2-phenylindole (DAPI) $(0.5$ $\left.\mathrm{mg} \mathrm{ml}^{-1}\right)$ and/or propidiumiodide $\left(1 \mu \mathrm{g} \mathrm{ml}^{-1}\right)$ in an antifade solution (Vectashield, Vector Laboratories). A Zeiss Axioskop fluorescence

Received 13 October 1993; accepted 4 January 1994.

1. Dutrillaux, B. Chromosomal evolution in primates: tentative phylogeny from Microcebus murinus to man. Hurn. Genet. 48, 251-314 (1979).

2. O'Brien, S.J., Seuânez, H.N. \& Womack, J.E. Mammalian genome organization: An evolutionary view. Ann. Rev. Genet. 22, 323-351 (1988).

3. O'Brien, S.J.\& Graves, J.A.M. Report of the committee on comparative gene mapping. Cytogenet. cell Genet. 58, 1124-1151 (1991).

4. O'Brien, S.J. et al. Anchored reference loci for comparative genomemapping in mammals. Nature Genet. 3, 103-112 (1993)

5. Wienberg. J., Stanyon, R., Jauch, A \& Cremer, T. Molecular cytotaxonomy of primates by chromosomal in situ suppression hybridization. Genomics 8 347-350 (1990).

6. Wienberg. J., Stanyon, R., Jauch, A. \& Cremer, T. Homologies in human and Mecaca fuscata chromosomes revealed by in situ suppression hybridization with human chromosome specific DNA libraries. Chromosome 101, 265270 (1992)

7. Lengauer, C., Wienterg, J., Cremer, T., Lüdecke, H.J. \& Horstehmke, B. Comparative chromosome band mapping in primates by in situ suppression hytridization of band specific DNA microlibraries. Hum. Evol. 6, 67-71 (1991).

8. Jauch, A. et al. Reconstruction of genomic rearrangements in great apes and gibbons by chromosome painting. Proc. natn. Acad. Scl. U.S.A. 89, 8611B615 (1992).

9. Luke, S. \& Verma, A.S. Origin of human chromosome 2. Natune Genet. 2, 11 (1992).

10. Stanyon, R. et at. Molecular and classical cytogenetic analyses demonstrate an apomorphic reciprocal chromosomal translocation in Gorilla gorlila. Am. J. phys. Anthrop. B8, 245-250 (1992).

11. Lichter, P., Cremer, T., Borden, J., Manuelidis, L. \& Ward, D.C. Delineation of Individual chromosomes in interphase and metaphase cells by in situ suppression hybridization using recombInant DNA libraries. Hum. Genet. 80, 224-234 (1988)

12. PInkel, D. et al. Fluorescence in situ hybridization with human chromosomespecific libraries: Detection of trisomy 21 and translocations of chromosome 4. Proc. natn. Acad. Sci. U.S.A. 85, 9138-9142 (1988).

13. Collins, C. et af. Construction and characterization of plasmid tibrarles enriched in sequences from single human chromosomes. Genomics 11, 997-1006 (1991)

14. Vooijs, M. et al. Libraries for each human chromosome, constructed from sorter-enriched chromosomes by using linker-adaptor PCR. Am. J. hum. Genet. 52, 586-597 (1993).

15. Lengauer, C. et at. Painting of defined chromosomal regions by in situ suppression hybridization of libraries from laser-microdissected chromosomes. Cytogenet. cell Genet. B6, 27-30 (1991).

16. Scherthan, H. Localization of the repetitive telomeric sequence (TTAGGG) in two muntjak species and implications for their karyotypic evolution. Orogenet cell Genet. 53, 115-117 (1990).

17. Arnason, U., Gullberg, A.\& Widegren, B. The complete nucleotide sequence of the mitochondrlai DNA of the fin whale, Baleonoptera physalus. J. molec Evol. 33, 556-568 (1991).

18. Ohno, S. Sex chromosomes and sex linked genes (Springer, Berlin, 1967)

19. Graves, J.A.M \& Watson, J.M. Mammallan sex chromosomes: Evolution of organization and function. Chromosoma 101, 63-68 (1991).

20. Liming, S., Yingying, Y. \& Xingsheng, D. Comparative cytogenetlc studies on the red muntjac, Chinese muntjac and their $F$, hybrids. Cytogenet. cell Genet. 26, 22-27 (1980).

21. Neitzel, H. Karyotypenevolution und deren Bedeutung fär de Speclationsprozess der Cerviden (Cervidae; Artiodactyla; Mammella). (PhO thesis, University of Berlin, 1982).

22. Copeland, N.G. etal. A genetic linkage map of the mouse: current appllcations and future prospects. Science 262, 57-66 (1993)

23. Holmquist, G.P. Evolution of chromosome bands: Molecular ecology of non coding DNA. \&. molec. Evol. 28, 469-486 (1989).

24. Bickmore, W.A. \& Sumner, A.T. Mammalian chromosome banding - an expression of genome organization. Trends Genet. 5, 144-148 (1989).

25. Morton, N.E. Parameters of the human genome. Proc. natn. Acad. Sci. U.S.A. 88, 7474-7476 (1991)

26. Levy, H.P., Schultz, A.A., Ordonez, J.V. \& Cohen, M.M. DNA content measurements and an improved idiogram for the indian muntiac. Cytometry microscope equipped with single band pass filters (Chroma Technology, Battleboro) were used for visualization of FITC, $\mathrm{Cy} 3$ and DAPI fluorescence. Microphotographs were recorded on Kodak Ektachrome 400 colour slide film.

\section{Acknowledgements}

We thank A, Jauch and H. Zankl for critical reading of the manuscript and J. Gray, (DMC, UCSF, San Francisco, California) for generously providing the human plasmid and linker adaptor PCR libraries and the mousechromosome 11 linker adaptor PCR library. We thankJ. Wienberg forstimulatingdiscussions on the potential of FISH and CGH techniques in studies of chromosome evolution. T.C. was supported by a grant from the Deutsche Forschungsgemeinschaft and L.F. was supported by a grant of the Landesgraduiertenförderung Rhld-Pfalz.

14, 362-368 (1993)

27. Ried, T. Baldini, A. Rand, T.C. \& Ward, D.C. Simultaneous visualization of seven different DNA probes by in situ hybridization using combinatoria fluorescence and digital imaging microscopy. Proc. natn. Acad. Sci. U.S.A. 89, 1388-1392 (1992).

28. Dauwerse, J.G., Wiegant, J., Raap, A.K., Breuning, M.H. \& van Ommen, G.J.B. Multiple colours by fluorescence in situ hybridization using ratiolabelled DNA probes create a molecular karyotype. Hum. molec. Genet. 1 593-598 (1992).

29. Lengauer, $C$. et al. Chromosomal bar codes produced by multicolour fluorescence in situ hybridization with multiple YAC clones and whole chromosome painting probes. Hum. molec. Genet. 2, 505-512 (1993)

30. Fled, T., Arnold, N. Ward, D.C. \& Wienberg J. Comparative high-resolution mapping of human and primate chromosomes by fluorescence in situ hybridization. Genomics 18, 381 (1993).

31. Yerie, M. ot al. Accurate chracterization of porcine bivariate flow karyotype by PCR and fluorescence in situ hybridisation. Genomics 16, 97-103 (1993).

32. Breneman JW et at. The development of chromosome-specific composite DNA probes for the mourse and their application to chromosome painting Chromosoma 102, 591-598 (1993).

33. Guan, X.Y.. Trent, J.M. \& Meltzer, P.S. Generation of band-specific painting probes from a singie microdissected chromosome. Hum. molec. Genet. 2 $1117-1121$ (1993)

34. Lima-de-Faria, A in Fundamentals of Medical Cell Biology (ed. Bittar, E.E.) 115-162 (JAI Press, Greenwich, Connecticut, 1991).

35. Lima-de-Faria, A., Mitelrnan, F., Blomberg. J. \& Pfeifer-Ohlson, S. Telomeric location of retroviral oncogenes in humans. Hereditas 114, 207-221 (1991)

36. Holmquist, G.P. Chromosome bands, their chromatin flavors, and their functional features. Am. J. hum. Genet. 51, 17-37 (1992).

37. Gouttert, $E$ et al. Generation of a chromosome-22-specific cDNA library as confirmed by FISH analysis. Hum.Genet, 92, 623-626 (1993)

38. Kaltionierni, A. et al. Comparative genomlc hybridization for moiecular analysis of solid tumors. Science 258, 818-821 (1992).

39. du Manoir, S. et et. Detection of complete gains and losses by comparative genomic in situ hybridization. Hum. Genet. 90,590-610 (1993).

40. Speicher, M.R. et al. Molecular cytogenetic anałysis of fixed, paraffin embedded solld tumours by comparative genomic hybridization after universal DNA-amplification. Hum. molec. Genet. 2, 1907-1914 (1993).

41. Hetzel, J. Livestock genome research on the march. Nature Genet. 4, 327328 (1993).

42. Nelson, S.F. et al Genome mismatch scanning: a new approach to genetic linkage mapping. Nature Genet. 4, 11-18 (1993).

43. Schriever-Schwemmer G. \& Adler 1.-D. A mouse stock with 38 chromosomes derived from the reciprocal translocation T(7;15)33Ad. Cytogenet. cell Genet. 64, 122-127 (1993)

44. Armason, U. \& Best, B.P. Phylogenetic relationships within the Mysticeti (whalebone whales) based upon studies of highly repetitive DNA in extant species. Hereditas 114, 263-269 (1991)

45. Klever M., Grond-Ginsbach C., Scherthan H. \& Schroeder-Kurt, T.M Chromosomal in situ suppression hybridisation after Giemsa banding. Hum. Genet. 86, 484-486 (1991).

46. Meyne, J., Ratliff, R.L. \& Moyzis, R.K. Conservation of the human telomere sequence (TTAGGG) n among vertebrates. Proc. natn. Acad. Sci. U.S.A. B6, 7049-7053 (1989)

47. Adegoke, J.A., Arnason, U. \& Wiedegren, B. Sequence organization and evolution, in all extant whalebone whales, of a DNA satelifte with terminal chromosome localization. Chromosoma 102, 382-388 (1993).

48. Pinket, D., Straume, T. \& Gray, J.W. Cytogenetic analysis using quantitative. high sensitivity, fluorescence hybridization. Proc. natn. Acad. Sci. U.S.A. 83, 2934-2938 (1986)

49. Scherthan, $H$. et af. Chromosomal in situ hybridization with double labelled DNA: signal amplification at the probe level Cytogenet. cell Genet. 60, 4-7 (1992).

50. Miashita, K et al. A mouse chromosome 11 library generated from sorter chromosomes using linker-adapter polymerase chain reaction. Cytogenet. cell Genet. 66, 4-7 (1994).

51. Evans, E.P. in Genetic variants and strains of the laboratory mouse (eds Lyon M.F. \& Searle A.G.) 576-581 (Oxford University Press, Oxford, 1984) 